\title{
ExeAnalyzer: A Deep Generative Adversarial Network for Multimodal Online Impression Analysis and Startup Funding Prediction
}

\author{
Kai Yang \\ Department of Information Systems \\ City University of Hong Kong \\ kayang6-c@my.cityu.edu.hk
}

\begin{abstract}
With the rise of equity crowdfunding platforms, entrepreneurs' online impressions are of great importance to startups' initial funding success. Guided by the design science research methodology, one contribution of our research is to design a novel Generative Adversarial Network, namely ExeAnalyzer, to analyze CEOs' online impressions by using multimodal data collected from social media platforms. More specifically, ExeAnalyzer can detect CEOs' first impressions, personalities, and other sociometric attributes. Based on a dataset of 7,806 startups extracted from AngelList, another contribution of our research is the empirical analysis of the relationship between CEOs' online impressions and startups' funding successes. Our empirical analysis shows that CEOs' impression of dominance is negatively related to startups' funding performance, while the social desirability of CEOs is positively associated with startups' funding success. Our empirical study also confirms that the impression features extracted by ExeAnalyzer have significant predictive power on startups' funding performance.
\end{abstract}

\section{Introduction}

The emergence of equity crowdfunding platforms, such as AngelList and Crunchbase, has benefited the startup entrepreneurs who need to raise money for the development of their business. On the one hand, entrepreneurs can make investors acquaintance online, without physical face-to-face communication. On the other hand, investors may also seek investment opportunities by following some potential entrepreneurs. On platforms such as AngelList or Crunchbase, most of the entrepreneurs disclose their personal social media accounts, such as Twitter, LinkedIn, and Facebook, so that the investors can easily access more information about the entrepreneurs. The management of the social media content presented to the

\author{
Raymond Y.K. Lau* \\ Department of Information Systems \\ City University of Hong Kong \\ raylau@cityu.edu.hk
}

investors is becoming increasingly important because the impression the investors have of the entrepreneurs is formed based on the information provided to them. Therefore, it is not surprising that the impression entrepreneurs convey online plays an important role in startups early success. However, although the formation and management of online impressions have increased in importance in recent years [25] [29], to our best knowledge, research on the firm-level impact of startup executives online impression is still a neglected area in entrepreneurial finance. A large-scale empirical study is needed to directly examine the impact of entrepreneurs online impression on startups early success in terms of financing performance. The difficulty lies in how to analyze the impression conveyed by executives across multimodal social media platforms in a more effective way. In the traditional methodology, human participants are required to rate social media information based on their subjective perception of the subjects personalities. This approach is time-consuming and costly, which prevents researchers from conducting a large-scale empirical analysis.

In this study, we propose a novel deep learning-based method, ExeAnalyzer, to comprehensively analyze the impression conveyed by executives online based on information available across multimodal social media platforms. With the development of deep learning methodologies in recent years, machines have been endowed with the ability to make human-like judgments. For instance, tasks, such as face detection or object detection, are now capable of remarkable performance and can outperform human judgments [9]. Thus, it is technically feasible to employ machine learning methodologies to analyze executives online impressions automatically to conduct a large-scale empirical analysis. Indeed, we have seen some early attempts to automatically recognize the first impression conveyed by human faces [7] [18]. Besides, some researchers have investigated the automatic detection of individuals personalities from their social media posts. We combine these early attempts in the proposed 
ExeAnalyzer to achieve a comprehensive analysis of the impression conveyed by executives online.

According to the literature on online impression formation, visual cues, such as profile images, as well as textual cues, such as their textual posts, are two of the important cues for forming impressions online ([22]). In addition, sociometric information (e.g., the number of friends) available on social platforms shape impressions as well ([32]). Therefore, ExeAnalyzer is designed to analyze the online impression from three types of information, namely, visual, textual, and sociometric information. Specifically, we utilize a novel first-impression predictor based on the GAN, which is an advanced deep learning approach, to predict the first impression conveyed by executives profile images. The proposed approach can identify four dimensions of first impressions: perceived age, perceived intelligence (IQ), perceived trustworthiness, and perceived dominance. These dimensions are selected because it has been reported that they are the comprehensive abstract qualities the perception of others is based on the work of McCurrie et al. [18]. As for the textual information, we utilize a deep bi-LSTM network to capture the personalities conveyed by executives textual posts. Moreover, based on the systemic functional linguistic theory (SFLT) introduced in [18], we use a lexicon-based method, LIWC, to extract linguistic cues covering all the three meta-functions (i.e., ideational, interpersonal, and textual) in the SFLT. Additionally, the ExeAnalyzer extracts and organizes sociometric information, such as the number of friends and the total number of favorites or replies. After processing information collected from the multimodal social platforms, the ExeAnalyzer will provide a comprehensive analysis report.

Empowered by the proposed ExeAnalyzer, we conduct a large-scale empirical analysis to examine the impact of the impression conveyed online by startup entrepreneurs on the success of startups at the early stage. Our dataset, which comes from AngelList, is composed of 7806 companies and 7056 CEOs. Similar to many previous studies [14] [10], we categorize the social media platforms into professional and non-professional, based on the users they have. We choose AngelList and LinkedIn to represent professional social platforms, and Twitter as the non-professional one. Then, we collect the CEOs social media data on these platforms. Specifically, by restricting the time to the startups early stage, we collect their historical profile images on AngelList, their education or experience-related attributes on both AngelList and LinkedIn, and historical social media posts and other sociometric attributes on Twitter. After extracting the impression conveyed by the data on multimodal social platforms using ExeAnalyzer, we employ econometric methodologies to explore the relation between executives online impression and startups early success. Consequently, we successfully verify that the impression features extracted by ExeAnalyzer are statistically related to firms success at the early stage in terms of financing performance. Moreover, we take a further step to develop a machine learning-based startup success prediction method and verified the predictive power of the features extracted by ExeAnalyzer.

Overall, the contributions of our work are four-fold. First, a novel deep learning-based impression analyzer, ExeAnalyzer, is proposed; it can analyze startup entrepreneurs online impressions via data from multimodal social media platforms. We design experiments to verify the effectiveness of the proposed IT artifact. Second, utilizing the proposed ExeAnalyzer, we conduct a large-scale empirical analysis to explore the relationship between executives online impressions and the startups success at the early stage. Third, we construct a machine learning-based approach to predict startups success, and successfully verify the predictive power of the impression features detected by ExeAnalyzer. To the best of our knowledge, we are among the first to verify the predictive power of executives online impressions over the success of startups at the early stage. Fourth, from a theoretical standpoint, our work advances data science knowledge and techniques, specifically in terms of executives impression analysis on social media. The managerial implication of our work is that using our design artifacts [13], startups can better understand the impressions conveyed by their top executives. The academic implication is that researchers can conduct empirical studies on a larger scale, using our design artifacts to measure executives online impressions.

\section{Related Work}

\subsection{Online Impression of Executives and Startups' Early Success}

In the field of business venturing, it is well known that high-tech startups are hard to survive without receiving funding from angel investors or venture capitals [4], and hence the ability of a startup company to raise funding is a key proxy to measure their early success [30] [5]. Recently, a new form of financing way, equity crowdfunding, is gaining more and more attention and becomes increasingly popular amount technology startups [21]. Accordingly, equity crowdfunding platforms like AngelList, Fundable, 
EquityNet, and Crunchbase ${ }^{1}$ have attracted a large group of entrepreneurs and investors. These platforms themselves are also social media that try to build up the social network between the startup entrepreneurs and investors. To gain more attention from investors, startups companies should actively engage in social media. Existing literature has examined that the active engagement of startup companies is highly correlated to their early success in raising funding [36]. Evidence provided by Liang and Yuan [15] shows that funding investors in Crunchbase are more likely to invest in a particular company if they have a stronger relationship in terms of closeness in the social network. Sharchilev et al. [28] utilize the general features (i.e., firms basic information), investor features, and people features (i.e., startup founders' social media information) to predict startup success. Besides, some other studies in this field try to use social media features in Crunchbase to recommend a portfolio of potential startups to investors [37]. Therefore, the potential of the startups can be inferred from their very early stage via behaviors of both startup companies and founders on social media. In the age of social media, many acquaintances and connections are made within these platforms, the first impression perceived by investors towards startup founders is plausible to make a difference in their final investment results. Despite the importance of founders perceived impression on social media, impression management of the startup founders is still a neglected segment of business venturing.

\subsection{Existing Impression Analytics Approaches}

The assessment of individuals social media impression is a subjective task, and human participants are needed for rating the social media information subjectively in traditional psychology literature. For example, in the work of Stuart and Abetti [30], 30 undergraduates were asked to rate the faces of CEOs in order to assess their impression. However, such type of method is time-consuming and it is impossible to conduct large-scale empirical studies to demonstrate the corporate-level impact of executives social media impression. With the development of machine intelligence, computers now are enabled to substitute humans for making human-like judgments. In the field of computer vision, tasks like object detection [20], face recognition [2] or even facial expression recognition [27] have already reached outstanding performance and some of them even outperform human

\footnotetext{
${ }^{1}$ URLs: https://angel.co/; https:/www.crunchbase.com/ https://www.fundable.com/; https://www.equitynet.com/;
}

judgments [9]. With the booming of deep learning technology, the trend that human is substituted by machine in some simple task is increasing. However, the task of impression detection is more subjective than traditional tasks like object detection. Is it possible for us to apply the novel machine learning techniques to conduct a comprehensive impression analysis based on top executives social media information?

McCurrie et al. [18] utilize a deep learning model to analyze individuals profile photos and predict their first impressions in terms of the following four dimensions: perceived intelligence, perceived trustworthiness, perceived age, perceived dominance. The reason to choose these four dimensions is that they can provide comprehensive abstract qualities for the perception of others [18]. Although there are some existing studies regarding solving the automatic impression prediction problem, compared to some other tasks like face detection or object detection, relatively less attention is located to it. As one of its challenges, the machine learning training process needs a labeled dataset, while the impression labels should be given by human raters and it is costly to obtain. Even if we have a small labeled dataset, it may not sufficient to train a deep learning model with a large number of parameters, and thus the model we trained may not be robust enough. We still need to find a trade-off between the cost of obtaining samples and the performance of the model.

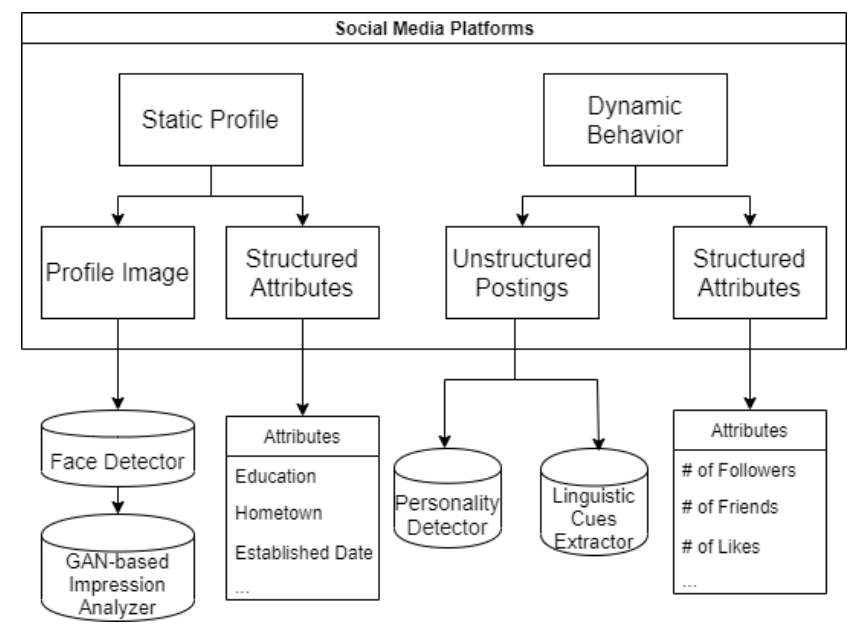

Figure 1. Overall Architecture of ExeAnalyzer

\section{ExeAnalyzer: Social Executives Impression Analyzer}

\subsection{Overall Architecture}

In this paper, we try to design a novel IT artifact, namely ExeAnalyzer, that can give a comprehensive 
analysis of startups CEOs' social media impression. The overall architecture is shown in Figure 1. As for a stranger, the first thing they see after entering CEOs personal pages is their profile information, such as profile image, and then their recent social activities like social media postings will be viewed. In online impression formation literature [22], visual cues, such as ones profile image, and textual cues has been reported important for humans to form their impression. Therefore, in the proposed ExeAnalyzer, we include two sources of social media information, namely, the static profile and dynamic social activities. Following the design science framework proposed by [1] which categorized computer-mediated communication (CMC) data as structural or un-structural, we categorize each source of social media data into structural and un-structural attributes. For the profile information, the profile images are un-structural and other attributes like education level or location are structural. For social media activities, the content of the postings is un-structural, and the numbers of responses and likes are structural. These structural data convey ones sociometric information, which is also essential for impression formation [32], and thus ExeAnalyzer should also have the ability to deal with them. Since the structural information is easy to extract, the challenges of the ExeAnalyzer lie in the processing of those un-structural data. Firstly, we utilize a novel GAN-based impression predictor to predict the perceived first impressions of CEOs faces in terms of four dimensions: intelligence, dominance, trustworthiness, and age. Note that the age here refers to their perceived age rather than their real age. Secondly, to analyze the social media postings created by CEOs, we use a deep learning-based method to uncover their big-five personalities according to their writing patterns. Thirdly, to extract more semantic information delivered by CEOs social media postings, we apply the Linguistic Inquiry and Word Count (LIWC) to analyze the texts. Finally, as for the structural attributes, we collect ones education level, location, endorse, skills, past job experiences, and investments from professional social platforms like Linkedin and AngelList. We collect the number of followers, friends, the total number of postings, likes, and replies in non-professional social platforms like Twitter. After the processing of both structural and un-structural data, we combine them together and produce a comprehensive analysis report.

\subsection{GAN-based Impression Predictor}

Generative adversarial networks (GANs) are a type of deep generative neural network that was introduced by [12]. Its algorithmic architecture includes two components: a generator and a discriminator. The generator is responsible to generate fake instances, while the discriminator is to discriminate these fake instances out of real samples. The two networks are set up in a contest or a game where the generator model aims to fool the discriminator model, and the discriminator is to increase the ability to detect fake images.

To predict ones first impression according to their faces, we use the dataset of [18] to train a deep learning-based model. This dataset incorporates 6300 images of faces with four dimensions first impressions labeled by humans: IQ, Age, Trustworthiness, and Dominance. The current state-of-the-art first impression predictor is based on CNN and is introduced in [18]. However, the existing CNN-based approaches have some limitations. The first challenge that previous studies have not solved is the lack of labeled samples. Specifically, training a deep learning model like CNN needs a lot of samples, and it is easy to overfit when the size of the samples is small. Since GANs have the ability to learn patterns about human faces from the unlabeled dataset while the unlabeled face images are relatively cheap on the internet, we argue that the GANs can provide more information for the impression prediction process if additional unlabeled face images can be considered. However, training a GAN needs a large volume of human faces samples and the CEO faces that we have now are not enough. We address this challenge by utilizing an open dataset, namely, Celeba [16], which contains 202,599 number of face images, together with the labeled first impression dataset and CEO faces dataset to train the GAN. As shown in Figure 2 , we have three datasets for the training process, while two of them are unlabeled and the other is with labels.

We follow the disposal of [19] and [26], which extend the original unsupervised GAN to solve the semi-supervised problem. Due to the stable performance of DCGAN in low-resolution human face generation [24], we develop our GAN-based impression predictor based on DCGAN. The setting of our generator is basically the same as that of DCGAN, while the difference lies in the discriminator. As shown in Figure 2, an input image goes through 4 layers of convolution layers first, and after each convolution layers, a batch normalization and ReLU operations are followed. After the last convolution layer, the network is divided into two branches: one goes across a fully connection (FC) layer and Sigmoid layer to indicate whether the image is fake; the other goes across several FC layers and has one-dimension output indicating the first impression score. The loss function of this model 
contains two parts: the supervised and unsupervised part:

$$
\begin{gathered}
\text { Loss }=L_{\text {unsupervised }}+L_{\text {supervised }} \\
L_{\text {unsupervised }}=E_{x \sim P_{\text {data }}}[\log (D(x))]+ \\
E_{z \sim N(0,1)}[1-\log (D(G(z)))] \\
L_{\text {supervised }}=\|y-R(x)\|
\end{gathered}
$$

Where $p_{\text {data }}$ is the real distribution of the image; $D(x)$ is the output of discriminator indicating whether the image is true; $\mathrm{z}$ is a list of random variables sampled from multinormal distribution; $R(x)$ is the regression output of the discriminator. For each iteration, we fix the parameters in the generator first and optimize the parameters in discriminator, and then fix the discriminator and optimize the generator.

However, a problem arises when we try to combine several data sources to train a GAN. We know that the task of the generator is to simulate the distribution of the data source fed into the GAN. Now that we have three sources that probably have a different distribution with each other (e.g., their gender and age distributions may be different), the generator may be misled and generate a distribution that is similar to none of the datasets. To alleviate this problem, we incorporate a sample selection process in the proposed GAN-based impression predictor. The intuitive idea is that we should provide more diversity faces from a domain-general dataset (e.g., Celeba) to ensure the network is fully trained in the early training stage, while after the generator can produce stable and diversity fake images, the data come from the domain-specific dataset (e.g., our labeled dataset and CEO faces dataset) can be applied to fine-tune the network. Once the GAN is trained to adapt the domain-specific datasets distribution, more patterns in the domain-specific dataset can be discovered, and hence the supervised learning process can be benefited. Accordingly, we utilize a weighted sampling method to merge different datasets together. Specifically, in each training iteration, we define the probability of choosing a domain-general dataset in iteration $i$ as $p_{i}^{(G)}$, the total iteration needed for training the model as $N$, the total number of the domain-specific dataset as $M$, the probability of choosing domain-specific dataset $\mathrm{m}$ in iteration $i$ as $p_{i}^{(m)}$. In the paper, we set up the following relationship when training the network: $p_{i}^{(G)}=1-\frac{i}{N}$ and $p_{i}^{(m)}=\frac{1-p_{i}^{(G)}}{M}$.

In the early training iteration, the domain-general samples have more probability to be chosen,
Table 1. Performance of the Proposed GAN-based Impression Predictor w.r.t R2

\begin{tabular}{lllll}
\hline & Trust. & Dom. & Age & IQ \\
\hline CNN-based & .38. & .46 & .72 & .24 \\
\hline Proposed Approach & .42 & .50 & .73 & .29 \\
\hline
\end{tabular}

and in the later iteration, the GAN is fed with more domain-specific samples to capture more domain-specific knowledge. In our case, the domain-general dataset is Celeba, and the domain-specific dataset is the labeled first impression dataset and unlabeled CEO faces dataset. We separate the first impression dataset into training and testing set following the disposal in [18], and we evaluate the performance of the model using R2. Finally, we find that the proposed GAN-based first impression predictor outperforms their CNN-based model in terms of all the four dimensions. The experimental results are shown in Table 1.

\subsection{Personality Detector and Linguistic Cues Extractor}

In the psychological trait theory, the Big Five personality model, also known as the five-factor model, provides a taxonomy for grouping personality traits [11]. Specifically, the theory identifies five factors: Extraversion (attention-seeking, sociable, playful vs. social withdrawal, shy), Neuroticism (helplessness, depressive, anxious vs. clam, fearlessness), Agreeableness (friendly, cooperative vs. deceitfulness, suspiciousness), Conscientiousness (self-disciplined vs. inefficient, rashness, careless), Openness (insightful, magical thinking, eccentricity vs. unimaginative, closed-minded). The technical feasibility of mining ones personalities based on their writings has been fully discussed in previous literature [17].

In this paper, we apply a deep Bi-LSTM network introduced by Yang and Lau [35] to extract CEOs big five personality traits. This type of network is regarded as having the ability to extract both semantic and grammatical information that reflect ones personality [23]. The deep Bi-LSTM personality detector will be trained by an open dataset, namely myPersonality [3], which consists of 10000 status updates from 250 Facebook users along with their big-five personality scores. By training and testing in the baseline dataset, we confirm that the performance of our constructed model is similar to that shown in [35]. In addition, to discover more abstract aspects of individuals' personality, we construct higher-order personality traits based on the big five models. Specifically, two 


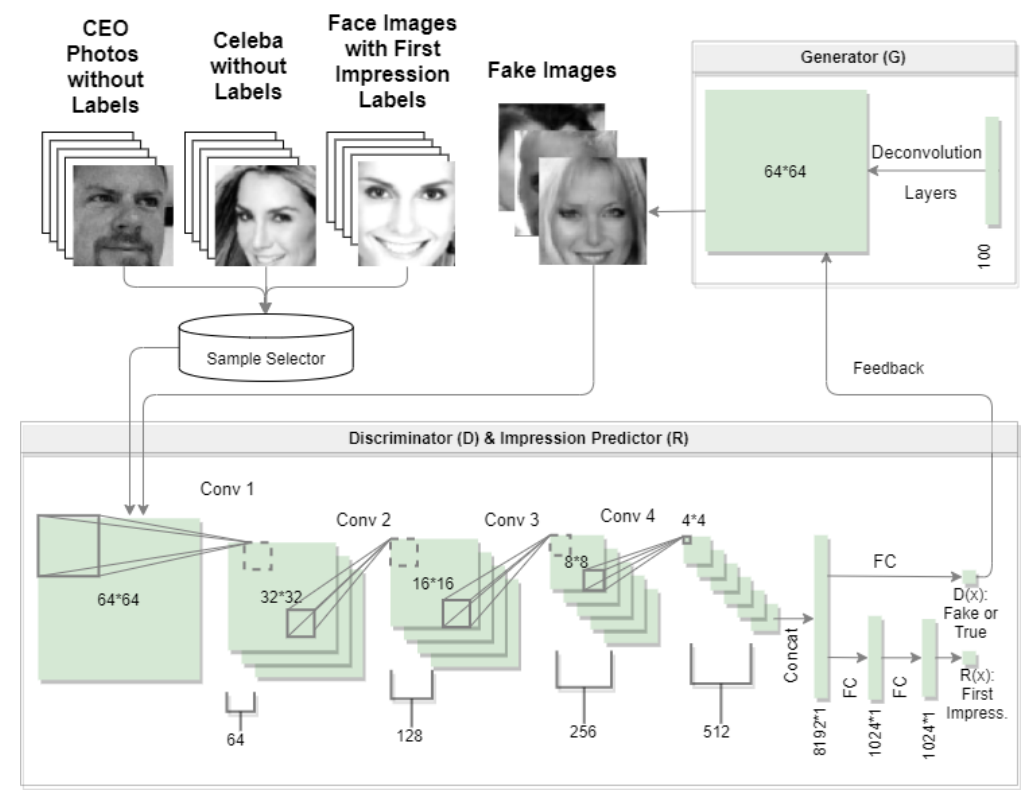

Figure 2. GAN-based Impression Predictor

higher-order traits, i.e., alpha and beta, are constructed following the methodology introduced by Digman [8] and DeYoung et al. [6], which are shown as follows.

$$
\begin{gathered}
\text { Alpha }=A G R+C O N-N E U \\
\text { Beta }=E X T+O P N
\end{gathered}
$$

Where EXT, CON, NEU, AGR, and OPN are the abbreviations of extraversion, conscientiousness, neuroticism, agreeableness, and openness respectively. Alpha represents a social desirability factor, while Beta is interpreted as plasticity.

In addition, we construct a linguistic cues extractor using Linguistic Inquiry and Word Count (LIWC) [31], which is a lexicon widely applied in natural language processing tasks. LIWC can not only extract linguistic features but also features reflecting ones affective processes (e.g., emotion), biological processes (e.g., health or body status), personal concerns (e.g., workor home-related), and so on. We apply all 90 categories in LIWC2015 as the output of linguistic cues extractor. Due to the limitation of space, please refer to Tausczik and Pennebaker [31] for the descriptions of LIWC outputs.

\section{Evaluation of ExeAnalyzer}

\subsection{Dataset Description}

We collect information about startup companies in AngelList, which is a popular equity crowdfunding platform. In AngelList, startups can set up their official page representing information like firms basic information, firms founders and current team, board members, investors, the total amount of each funding round, etc. Since AngelList also includes many venture capital firms which we are not interested in for this study, we only select the companies which are noted as startup by the platform. After filtered by the above conditions, we totally collect 7086 records from AngelList. We find that some of the funding information recorded by AngelList is missing, so we search their funding records in Crunchbase, which is another popular crowdfunding platform, and merge them into our dataset. Totally, 3257 startups get funding recorded supplemented from Crunchbase. For each firm, we collect the founders AngelList personal page and get a total of 13241 records. If a firm has multiple founders, we only select the most representative one (e.g., those who have the CEO title) to represent the firms. Since some of the firms do not have founders records, there are 7056 startups CEOs left. Then, we collect information from the firms official Twitter account, the founders personal Twitter account, and personal AngelList pages if they have.

Totally, we get 7056 founders AngelList accounts and 3169 Twitter accounts. As for the founders Twitter accounts, we obtain their established date and filter out those created later than the date that the startup received their first funding. The reason for this disposal is that we try to utilize the proposed ExeAnalyzer to extract social media features to predict the firms performance in raising funding, and hence we should make sure 
Table 2. Descriptive Statistics

\begin{tabular}{llllll}
\hline Variable & Obs & Mean & Std.Dev. & Min & Max \\
\hline Trust. & 2150 & .385 & .055 & .189 & .589 \\
Dominance & 2150 & .424 & .062 & .214 & .638 \\
IQ & 2150 & 4.01 & .239 & 3.05 & 4.802 \\
Age & 2150 & 4.115 & .584 & 1.475 & 6.215 \\
Alpha & 2636 & 1.222 & .311 & -.007 & 1.92 \\
Beta & 2636 & 1.094 & .243 & 0 & 1.957 \\
\#Investment & 7056 & 2.843 & 11.809 & 0 & 248 \\
\#Reference & 7056 & .424 & 1.333 & 0 & 24 \\
IS_PHD & 7056 & .009 & .094 & 0 & 1 \\
IS_MBA & 7056 & .033 & .178 & 0 & 1 \\
\#Experience & 7056 & 5.539 & 38.955 & 0 & 944 \\
Log(\#Google) & 7806 & .961 & 2.771 & 0 & 17.545 \\
Log(Total) & 7806 & 13.643 & 2.211 & 0 & 24.009 \\
Log(First) & 7806 & 12.732 & 1.934 & 0 & 21.212 \\
Log(\#favor) & 2636 & 4.735 & 4.709 & 0 & 16.783 \\
Log(\#Tweet) & 2636 & 3.045 & 2.735 & 0 & 7.298 \\
Log(\#retweem) & 2636 & 5.272 & 4.976 & 0 & 16.463 \\
Log(\#response) & 2636 & 3.411 & 3.634 & 0 & 13.783 \\
\hline
\end{tabular}

that the information we use is available at the time the startups had not received funding. Eventually, we find that 533 founders Twitter accounts are created after the startup finished the first round, so we exclude them from our dataset and finally get 2636 accounts. Since CEOs profile images in AngelList may have been changed over time, the one we see now may not the one that investors viewed in the early stage of the startups. Therefore, it may be inaccurate if we analyze the first impressions according to the profile images we obtain now. To alleviate this problem, we collect the historical profile images of CEOs through the Archive, which is a non-profit digital library containing the historical versions of a large volume of web pages. We track back the historical versions of each CEOs AngelList webpages. We argue that two years before and after the first round can be regarded as the early stage of the startup, and we collect the CEOs historical profile images which had been collected by archive during this period. Totally, we get 2150 CEOs historical profile images and utilize the proposed ExeAnalyzer to get their perceived first impressions. The descriptive statistics of our collected dataset is reported in Table 2.

\subsection{Econometric Analysis}

To verify that the impression features extracted by ExeAnalyzer are predictive for startups financing ability, we conduct an empirical study using the econometric methodology based on the dataset we collected. We are interested in startups early success in terms of their ability to attract venture capitals. Our hypothesis is:

H1: Features detected by ExeAnalyzer is predictable for startups early financing success.

Accordingly, to get a robust result, we have the
Table 3. Effects of Executives Impression on Raising Funding in Equity Crowdfunding Platforms

\begin{tabular}{|c|c|c|}
\hline & $\begin{array}{l}\text { (1) } \\
\text { Log(First)- } \\
\text { AngelList }\end{array}$ & $\begin{array}{l}\text { (2) } \\
\text { Log(Total)- } \\
\text { AngelList }\end{array}$ \\
\hline Trustworthiness & $\begin{array}{l}-0.4066 \\
(-0.5185)\end{array}$ & $\begin{array}{l}0.1985 \\
(0.2394)\end{array}$ \\
\hline Dominance & $\begin{array}{l}-2.1236 * * * \\
(-2.9265)\end{array}$ & $\begin{array}{l}-1.9216^{* *} \\
(-2.5034)\end{array}$ \\
\hline IQ & $\begin{array}{l}0.3572 * \\
(1.9346)\end{array}$ & $\begin{array}{l}0.1983 \\
(1.0150)\end{array}$ \\
\hline AGE & $\begin{array}{l}0.0374 \\
(0.4956)\end{array}$ & $\begin{array}{l}0.0367 \\
(0.4591)\end{array}$ \\
\hline Firm-level Controls & YES & YES \\
\hline CEO-level Controls & YES & YES \\
\hline _cons & $\begin{array}{l}13.1529 * * * \\
(9.4116)\end{array}$ & $\begin{array}{l}13.8089^{* * * *} \\
(9.3410)\end{array}$ \\
\hline $\mathrm{N}$ & 2098 & 2098 \\
\hline R2 & 0.186 & 0.180 \\
\hline
\end{tabular}

following two dependent variables measuring startups early success: 1) $\log$ (First), the logarithm of the amount of first funding round; 2) $\log$ (Total), the logarithm of total funding amount within 5 years after receiving the first round. We estimate the following regression:

$$
\begin{gathered}
Y=\alpha+\beta X+\delta \text { Control }+ \\
\text { Year }+ \text { Market }+ \text { Location }
\end{gathered}
$$

Where $\mathrm{X}$ includes the independent variables of interest. We separate the independent variables into two sets, one comes from CEOs professional social media, i.e., AngelList, and the other comes from the CEOs non-professional social media, i.e., Twitter. As for the AngelList features, we analyze CEOs AngelList profile images and have the four dimensions first impression features: Trustworthiness, Dominance, IQ, and AGE. Note that the IQ and AGE here are the perceived IQ and perceived AGE rather than their real ones. As for the Twitter features, we include the higher-order factors of personality, namely, Alpha and Beta. Unlike AngelList where CEOs tend to use their own pictures, we find that nearly half of the CEOs do not use their own pictures as their profile photos. Instead, they use a cartoon, scenery pictures, their kids pictures, etc. Thus, we cannot use the proposed first impression predictor to analyze their first impression, as we are hard to know whether the faces detected from their Twitter profile pictures belong to themselves. Besides, some structural features in Twitter are also included as independent variables. $\log$ (favor) and $\log$ (tweet) represent the number of CEOs tweets and favorites received by others. We do not include the number of replies and retweets since we find that they are highly correlated to the number of favorites and will cause a multicollinearity problem. Since the output of 
linguistic cues generated by LIWC according to CEOs Tweets contains 100 dimensions, we do not include the linguistic cues due to the limitation of space. We will examine their predictive power in the next section.

As for the education information (IS_PHD and IS_MBA) and experience (\#Experience), we merge the data collected from Linkedin and AngelList together. Besides, in AngelList, we collect the number of investments launched by the CEOs (\#Investment) to see whether the CEOs themselves are investors. Applying our personality detector, we get the big-five personality traits of CEOs. To obtain more comprehensible results toward CEOs personality, we following the disposal of [8] to construct higher-level factors based on the big-five personality. Two higher-order factors are constructed: Alpha and Beta. For the firm-level features, we collect data from their official Twitter accounts and extract some fundamental features like the number of tweets, retweets, likes, and favorites. Besides, previous literature has verified the importance of corporate reputation and corporate financial performance [33], so we also construct a variable to evaluate startups reputation. To measure it, Weng and Chen [33] use the frequency of firms mentioned by several main-stream newspapers as the proxy. However, as for startups, we are hard to find their names in main-stream media. Therefore, we try to use the number of results searched by Google giving their companies full names. To ensure the results are available before the first round of the startup, we set a time filter in Google to only include historical records. In addition, we collect some basic information about the startups, such as their location and the market they belong to.

We include both firm-level and individual-level controls in the regression model. As for the firm-level controls, we have $\log (\#$ Google), $\log$ (\#firm_Tweet), Log(\#firm_favor), Has_facebook(Firm), has_blog(Firm). Besides, the year that first-round happened, the market (come from AngelLists market label), and the location of the startups are also included as controls. As for the CEO-level controls, we have \#Reference, IS_PHD, IS_MBA, \#past job, and \#Investment(CEO).

The empirical results are shown in Table 2 and Table 3 which are grouped by four regression models. Models 1-2 in Table 2 try to find out the effects of CEOs first impression on AngelList on the startups early success, while the Models 3-4 in Table 3 try to find out the effects of CEOs non-professional social media impression on startups success. We find that perceived Dominance is significantly and negatively related to the amount of funding they can raise. Besides, we also find that CEOs who present higher social desirability (Alpha) in their Twitter postings are more likely to raise more money. As
Table 4. Effects of Executives Impression on Raising Funding in Public Social Media

\begin{tabular}{|c|c|c|}
\hline & $\begin{array}{l}\text { (3) } \\
\text { Log(First) } \\
\text { Twitter }\end{array}$ & $\begin{array}{l}\text { (4) } \\
\text { Log(Total) } \\
\text { Twitter }\end{array}$ \\
\hline Alpha & $\begin{array}{l}1.5190 * * * \\
(8.7604)\end{array}$ & $\begin{array}{l}1.7606 * * * \\
(9.0366)\end{array}$ \\
\hline Beta & $\begin{array}{l}-0.1843 \\
(-0.6578)\end{array}$ & $\begin{array}{l}0.0599 \\
(0.1902)\end{array}$ \\
\hline Firm-level Controls & YES & YES \\
\hline CEO-level Controls & YES & YES \\
\hline _cons & $\begin{array}{l}12.8232 * * * \\
(6.8862)\end{array}$ & $\begin{array}{l}15.5868 * * * \\
(7.4492)\end{array}$ \\
\hline $\mathrm{N}$ & 2393 & 2393 \\
\hline $\mathrm{R} 2$ & 0.221 & 0.218 \\
\hline
\end{tabular}

for their social media activities, we find that the number of tweets they post does not significantly relate to the amount of funding. Instead, the number of favorites they received has a significant impact. This indicates that CEOs should care about the content they post rather than just post a lot.

\section{Prediction of Startups Early Success in Financing Performance}

Since we have verified that some of the features extracted by the proposed ExeAnalyzer are significantly related to the amount of funding the startups can raise, in this section, we step forward and examine the predictive power of these features on startups early financing success. We measure the early success by the amount of startups first-round or the total amount they raise within 5 years after the first round. The reason for this measurement is that funding has been reported as one of the important factors for the survival of startup companies ([4]). We conduct a series of experiments to test the predictive power of different types of features. Accordingly, we separate the features given by ExeAnalyzer into two sets: AngelList-based features and Twitter-based features.

For each feature set, we divide the whole dataset into two parts: $90 \%$ as the training set and $10 \%$ as the testing set. Several popular machine learning methods are chosen for the prediction task, including random forest regression (RFR), gradient boosting decision tree regression (GBDT), and boosting regression (BR). These ensemble-based methods have been proven useful in both academic and industry. For each set of experiment, we run the ensemble-based method twice: one only using control features without the feature set of interest (the Without column in Table 3), and the other use both control features and the feature set (the With column in Table 3). For each model, we use 
Table 5. Prediction Results and Comparison

\begin{tabular}{|c|c|c|c|c|c|c|c|c|}
\hline \multirow{2}{*}{ Feature Set } & \multicolumn{4}{|c|}{ Log(First) -R2 } & \multicolumn{4}{|c|}{ Log(Total) R2 } \\
\hline & Models & Without & With & Statistical Comparison & Models & Without & With & Statistical Comparison \\
\hline \multirow[t]{2}{*}{ AngelList } & RFR & 0.04 & 0.06 & $\mathrm{w}=8, \mathrm{p}=0.02$ & RFR & 0.10 & 0.11 & $\mathrm{w}=22, \mathrm{p}=0.31$ \\
\hline & GBDT & 0.06 & 0.06 & $\mathrm{w}=0, \mathrm{p}<0.00$ & GBDT & 0.11 & 0.11 & $\mathrm{w}=22, \mathrm{p}=0.31$ \\
\hline (Profile Images) & BR & 0.05 & 0.05 & $\mathrm{w}=17, \mathrm{p}=0.16$ & BR & 0.10 & 0.10 & $\mathrm{w}=21, \mathrm{p}=0.28$ \\
\hline \multirow{3}{*}{ Twitter (Personality) } & RFR & 0.02 & 0.12 & $\mathrm{w}=4, \mathrm{p}=0.01$ & RFR & 0.03 & 0.11 & $\mathrm{w}=5, \mathrm{p}=0.01$ \\
\hline & GBDT & 0.04 & 0.12 & $\mathrm{w}=6, \mathrm{p}=0.01$ & GBDT & 0.05 & 0.09 & $\mathrm{w}=3, \mathrm{p}<0.00$ \\
\hline & BR & 0.00 & 0.11 & $\mathrm{w}=4, \mathrm{p}=0.01$ & BR & 0.03 & 0.11 & $\mathrm{w}=5, \mathrm{p}=0.01$ \\
\hline \multirow{3}{*}{ Twitter-LIWC } & RFR & 0.02 & 0.10 & $\mathrm{w}=6, \mathrm{p}=0.01$ & RFR & 0.02 & 0.09 & $\mathrm{w}=9, \mathrm{p}=0.03$ \\
\hline & GBDT & 0.04 & 0.11 & $\mathrm{w}=0, \mathrm{p}<0.00$ & GBDT & 0.07 & 0.11 & $\mathrm{w}=5, \mathrm{p}=0.01$ \\
\hline & BR & 0.03 & 0.11 & $\mathrm{w}=2, \mathrm{p}<0.00$ & BR & 0.05 & 0.10 & $\mathrm{w}=6, \mathrm{p}=0.01$ \\
\hline \multirow{3}{*}{ ALL Features } & RFR & 0.03 & 0.14 & $\mathrm{w}=5, \mathrm{p}=0.01$ & RFR & 0.04 & 0.13 & $\mathrm{w}=3, \mathrm{p}<0.00$ \\
\hline & GBDT & 0.05 & 0.15 & $\mathrm{w}=0, \mathrm{p}<0.00$ & GBDT & 0.06 & 0.14 & $\mathrm{w}=1, \mathrm{p}<0.00$ \\
\hline & BR & 0.01 & 0.15 & $\mathrm{w}=3, \mathrm{p}<0.00$ & BR & 0.04 & 0.12 & $\mathrm{w}=8, \mathrm{p}=0.02$ \\
\hline
\end{tabular}

Note: One-tailed Wilcoxon signed-rank test is applied to compare the performance of experimental groups with and without the corresponding feature set

ten-fold cross-validation and get 10 sets of results. The experimental results are shown in Table 3, where the With and Without columns report the average R2 of these 10 sets. To statistically prove that the models with features of interest outperform those without, we apply the one-tailed Wilcoxon signed-rank test to compare the performance of two sets of experimental results [34]. From the experimental results, we find that by adding most of the feature set we choose, it can significantly improve the performance of the machine learning models. By adding all the features into the models, they get significant improvement, and the highest R2 reaches 0.15 in GBDT and BR when predicting the first round amount and 0.14 in GBDT when predicting the total amount. These experiments prove that the features extracted by the ExeAnalyzer have predictive power on firms early success.

\section{Conclusions}

Guided by the design science research methodology, one of the contributions of our research is the design of a novel IT artifact, namely ExeAnalyzer, to analyze executives online impressions based on multimodal social media data. The merit of ExeAnalyzer is that it can deeply analyze complex visual, textual, and sociometric data. Empowered by the proposed ExeAnalyzer, another main contribution of our research is to conduct a large-scale empirical analysis to examine the possible relationship between executives online impressions and startups funding success. This can also be seen as a demonstration of the utility of the proposed design artifact. Our empirical analysis shows that CEOs impress of dominance is negatively related to startups funding performance. Besides, CEOs who can demonstrate a higher level of social desirability are likely to raise more funding for their firms. Finally, our empirical study confirms that the features extracted by ExeAnalyzer can significantly improve prediction performance with respect to startups funding amounts. However, as one of our limitations, the causality between executives first impressions and the firms financing performance is still unclear. In future work, we will introduce more econometric methodologies to further examine the causality issue.

\section{Acknowledgement}

Our research work was partly supported by grants from the Research Grants Council of the Hong Kong Special Administrative Region, China (Projects: CityU 11525716 and CityU 11507219), the NSFC Basic Research Program (Project No. 71671155), and the CityU Shenzhen Research Institute.

\section{References}

[1] Ahmed Abbasi and Hsinchun Chen. CyberGate: a design framework and system for text analysis of computer-mediated communication. Mis Quarterly, pages 811-837, 2008. ISSN 0276-7783.

[2] Vicki Bruce and Andy Young. Understanding face recognition. British journal of psychology, 77(3): 305-327, 1986. ISSN 0007-1269.

[3] Fabio Celli, Fabio Pianesi, David Stillwell, and Michal Kosinski. Workshop on computational personality recognition: Shared task. In Seventh International AAAI Conference on Weblogs and Social Media, 2013.

[4] Annalisa Croce, Massimiliano Guerini, and Elisa Ughetto. Angel Financing and the Performance of HighTech StartUps. Journal of Small Business Management, 56(2):208-228, 2018. ISSN 0047-2778.

[5] Dominik Dellermann, Nikolaus Lipusch, Philipp Ebel, Karl Michael Popp, and Jan Marco Leimeister. Finding the unicorn: Predicting early stage startup success through a hybrid intelligence method. 2017.

[6] Colin G DeYoung, Jordan B Peterson, and Daniel M Higgins. Higher-order factors of the Big Five predict conformity: Are there neuroses of health? Personality 
and Individual differences, 33(4):533-552, 2002. ISSN 0191-8869.

[7] Abhinav Dhall and Jesse Hoey. First impressions-predicting user personality from twitter profile images. In International Workshop on Human Behavior Understanding, pages 148-158. Springer, 2016.

[8] John M Digman. Higher-order factors of the Big Five. Journal of personality and social psychology, 73(6): 1246, 1997. ISSN 1939-1315.

[9] Samuel Dodge and Lina Karam. A study and comparison of human and deep learning recognition performance under visual distortions. In 2017 26th international conference on computer communication and networks (ICCCN), pages 1-7. IEEE, 2017. ISBN 1509029915.

[10] Neha Garg and Rinkle Rani. Analysis and visualization of Twitter data using k-means clustering. In 2017 International Conference on Intelligent Computing and Control Systems (ICICCS), pages 670-675. IEEE, 2017. ISBN 1538627450.

[11] Lewis R Goldberg. An alternative" description of personality": the big-five factor structure. Journal of personality and social psychology, 59(6):1216, 1990.

[12] Ian Goodfellow, Jean Pouget-Abadie, Mehdi Mirza, Bing Xu, David Warde-Farley, Sherjil Ozair, Aaron Courville, and Yoshua Bengio. Generative adversarial nets. In Advances in neural information processing systems, pages 2672-2680, 2014.

[13] Alan R Hevner, Salvatore T March, Jinsoo Park, and Sudha Ram. Design science in information systems research. MIS quarterly, pages 75-105, 2004. ISSN 0276-7783.

[14] Ilkay Karaduman. The effect of social media on personal branding efforts of top level executives. Procedia-social and behavioral sciences, 99:465-473, 2013. ISSN 1877-0428.

[15] Yuxian Eugene Liang and Soe-Tsyr Daphne Yuan. Predicting investor funding behavior using crunchbase social network features. Internet Research, 2016.

[16] Ziwei Liu, Ping Luo, Xiaogang Wang, and Xiaoou Tang. Large-scale celebfaces attributes (celeba) dataset. Retrieved August, 15:2018, 2018.

[17] François Mairesse, Marilyn A Walker, Matthias R Mehl, and Roger K Moore. Using linguistic cues for the automatic recognition of personality in conversation and text. Journal of artificial intelligence research, 30: 457-500, 2007. ISSN 1076-9757.

[18] Mel McCurrie, Fernando Beletti, Lucas Parzianello, Allen Westendorp, Samuel Anthony, and Walter J Scheirer. Predicting first impressions with deep learning. In 2017 12th IEEE International Conference on Automatic Face \& Gesture Recognition (FG 2017), pages 518-525. IEEE, 2017. ISBN 1509040234.

[19] Augustus Odena. Semi-supervised learning with generative adversarial networks. arXiv preprint arXiv:1606.01583, 2016.

[20] Wanli Ouyang, Ping Luo, Xingyu Zeng, Shi Qiu, Yonglong Tian, Hongsheng Li, Shuo Yang, Zhe Wang, Yuanjun Xiong, and Chen Qian. Deepid-net: multi-stage and deformable deep convolutional neural networks for object detection. arXiv preprint arXiv:1409.3505, 2014.

[21] Jeannette Paschen. Choose wisely: Crowdfunding through the stages of the startup life cycle. Business Horizons, 60(2):179-188, 2017. ISSN 0007-6813.

[22] Ayellet Pelled, Tanya Zilberstein, Alona Tsirulnikov, Eran Pick, Yael Patkin, and Nurit Tal-Or. Textual primacy online: Impression formation based on textual and visual cues in Facebook profiles. American Behavioral Scientist, 61(7):672-687, 2017. ISSN 0002-7642.
[23] Matthew E Peters, Mark Neumann, Mohit Iyyer, Matt Gardner, Christopher Clark, Kenton Lee, and Luke Zettlemoyer. Deep contextualized word representations. arXiv preprint arXiv:1802.05365, 2018.

[24] Alec Radford, Luke Metz, and Soumith Chintala. Unsupervised representation learning with deep convolutional generative adversarial networks. arXiv preprint arXiv: 1511.06434, 2015.

[25] Giulia Ranzini and Elles Hoek. To you who (I think) are listening: Imaginary audience and impression management on Facebook. Computers in Human Behavior, 75:228-235, 2017. ISSN 0747-5632.

[26] Mehdi Rezagholiradeh and Md Akmal Haidar. Reg-gan: Semi-supervised learning based on generative adversarial networks for regression. In 2018 IEEE International Conference on Acoustics, Speech and Signal Processing (ICASSP), pages 2806-2810. IEEE, 2018. ISBN 1538646587.

[27] Caifeng Shan, Shaogang Gong, and Peter W McOwan. Facial expression recognition based on local binary patterns: A comprehensive study. Image and vision Computing, 27(6):803-816, 2009. ISSN 0262-8856.

[28] Boris Sharchilev, Michael Roizner, Andrey Rumyantsev, Denis Ozornin, Pavel Serdyukov, and Maarten de Rijke. Web-based startup success prediction. In Proceedings of the 27th ACM International Conference on Information and Knowledge Management, pages 2283-2291, 2018.

[29] Andra Siibak. Constructing the self through the photo selection-visual impression management on social networking websites. Cyberpsychology: Journal of psychosocial research on cyberspace, 3(1), 2009. ISSN 1802-7962.

[30] Robert Stuart and Pier A Abetti. Start-up ventures: Towards the prediction of initial success. Journal of business venturing, 2(3):215-230, 1987. ISSN 0883-9026.

[31] Yla R Tausczik and James W Pennebaker. The psychological meaning of words: LIWC and computerized text analysis methods. Journal of language and social psychology, 29(1):24-54, 2010.

[32] Stephanie Tom Tong, Brandon Van Der Heide, Lindsey Langwell, and Joseph B Walther. Too much of a good thing? The relationship between number of friends and interpersonal impressions on Facebook. Journal of computer-mediated communication, 13(3):531-549, 2008. ISSN 1083-6101.

[33] Pei-Shih Weng and Wan-Yi Chen. Doing good or choosing well? Corporate reputation, CEO reputation, and corporate financial performance. The North American Journal of Economics and Finance, 39: 223-240, 2017. ISSN 1062-9408.

[34] R F Woolson. Wilcoxon signedrank test. Wiley encyclopedia of clinical trials, pages 1-3, 2007.

[35] Kai Yang and Raymond Lau. Detecting Senior Executives' Personalities for Predicting Corporate Behaviors: An Attention-based Deep Learning Approach. 2019.

[36] Qizhen Zhang, Tengyuan Ye, Meryem Essaidi, Shivani Agarwal, Vincent Liu, and Boon Thau Loo. Predicting startup crowdfunding success through longitudinal social engagement analysis. In Proceedings of the 2017 ACM on Conference on Information and Knowledge Management, pages 1937-1946, 2017.

[37] Hao Zhong, Chuanren Liu, Junwei Zhong, and Hui Xiong. Which startup to invest in: a personalized portfolio strategy. Annals of Operations Research, 263 (1-2):339-360, 2018. ISSN 0254-5330. 